\title{
A theoretical account of the effects of environmental context upon cognitive processes
}

\author{
SARA J. NIXON and N. JACK KANAK \\ University of Oklahoma, Norman, Oklahoma
}

\begin{abstract}
Although the environmental context effect typically refers to superior performance in the context of original learning, this result is not consistently found across all experimental paradigms or instructional sets. Thus far, a precise statement of the underlying mechanisms and boundaries of the context effect has been absent from the literature, with the research having focused primarily on the robustness of the effect. The current exposition of a theoretical model and interpretation of the environmental context effect not only provides an explanation of the same-context testing superiority, but also provides the necessary parameters allowing for the prediction of situations in which same-context testing should not be superior. Furthermore, the model has implications for complex learning situations which have not been tested in the research literature. The current model is grounded in the principles of classical conditioning. Thus, it is assumed that environmental context stimuli function in the same way that classically conditioned stimuli do and, as such, have effects on the various phenomena and processes associated with classical conditioning (e.g., acquisition, transfer, extinction and spontaneous recovery, and latent inhibition). Furthermore, the saliency of such stimuli should be subject to boundaries analogous to those affecting other conditioned stimuli.
\end{abstract}

The environmental context effect, representing superior memory for information learned and tested in the same as opposed to different environments, has been accorded theoretical significance throughout the history of modern experimental psychology (e.g., McGeoch, 1942; McGovern, 1964). Numerous recent experiments have provided empirical support for the effect in both human (e.g., Block, 1982; Godden \& Baddeley, 1975; Hintzman, Block, \& Summers, 1973; Smith, 1979, 1982; Smith, Glenberg, \& Bjork, 1978) and animal (e.g., Jobe, Mellgren, Feinberg, Littlejohn, \& Rigby, 1977) research. However, the superiority of same-context testing has not been found across all experimental paradigms (e.g., recall vs. recognition) or instructional sets (see Block, 1982; Nixon \& Kanak, 1981; Smith et al., 1978), indicating that the underlying mechanism or mechanisms governing contextual associations have specifiable boundaries of sensitivity. Most attempts to explain the context effect have been more descriptive than explanatory, and some appear incapable of accounting for all of the available empirical data, especially those explanations that specify a strong "strategy" interpretation. Furthermore, most hypotheses do not account for the superiority of "different" context environments in tasks involving proactive inhibition (PI) (e.g., Dallet \& Wilcox, 1968) or retroactive inhibition (RI) (e.g., Bilodeau \& Schlosberg, 1951; Eckert, Kanak, \& Stevens, 1984; Greenspoon \& Ranyard, 1957).

Wickens, Tuber, and Wickens (1983) demonstrated im-

This article is based, in part, on a doctoral dissertation submitted by the senior author to the Graduate College of the University of Oklahoma. Requests for reprints should be addressed to Sara J. Nixon, Room 211 OMU, University of Oklahoma, Norman, OK 73019. portant parallels between animal memory for a conditioned response as a function of environmental context manipulations and a broad set of literature and theory in human learning and memory, with regard to PI effects. Although developed independently, the present theory is consistent with the efforts of Wickens et al. in supporting the law of parsimony by providing a theoretical account of environmental context effects based upon analogues of principles of classical conditioning and traditional associative theory. The theory not only defines the mechanisms, and their interactions, that govern the development and degree of influence of contextual associations, but also can be extended in a straightforward manner, through the principles of interference theory (see Postman \& Underwood, 1973), to account for and predict conditions under which "different" contexts produce superior performance.

We propose that the context effect occurs as a result of (1) incidental associations formed between general contextual stimuli and the list items and/or the list as a whole during acquisition, and (2) the contextual stimuli automatically activating and tending to elicit the list responses or items when the same contextual stimuli are physically present, or are reconstructed, during a retention test. Within this conception, by analogy, environmental context stimuli function as do classically conditioned stimuli in that they elicit response items qua responses and/or correct responses to the extent that they are actually predictive of differential reinforcement (see Mackintosh, 1975). Thus, contextual stimuli make list items "more available" and/or make more list items "availble" at above-threshold level, with context/list-item associative strength combining with list specific-item, or 
pair, associative strength. In free-recall tasks, either of these possible elicitation functions affecting "availability" is analogous to the functions of classically conditioned stimuli, since it is usually the "quantity" of responses that is measured. On the other hand, in pairedassociate (PA) learning, in which pairwise correctness of responding is typically the issue, contextual associations with the items serve to mediate the elicitation, and hence the availability, of the elements of the pair. The effect of context on stimulus-item functions in PA tasks should be most sensitive when conditions of acquisition or testing require stimulus-item "recall," as opposed to stimulus "recognition, since the elicitation function of contextual stimuli is most powerful in affecting "availability," which is at issue in recall but not in recognition. Contextual stimuli, however, may affect recognition memory through their effects upon generalization and differentiation processes. Thus, it is predicted that contextual stimuli will affect learning and memory in a manner analogous to that of other conditioned stimuli and, as such, contextual stimuli should have predictable effects on the phenomena and processes involved in acquisition, transfer, "unlearning," spontaneous recovery, and latent inhibition.

The exposition of the theory here will relate only to acquisition of context-item associations within a PA task, although the extensions of the influence of context-item associations to topics cited above are straightforward. Space does not permit a further elaboration of the theory to account for the effects of contextual associations on free-recall learning, classical serial learning, or recognition memory tasks. The PA task was chosen because (1) it has a substantial empirical and theoretical literature that allows precise predictions to be made, and (2) it employs readily differentiated stimulus and response components that are relevant to the general thesis of the model, an important factor for the test of a model grounded in the processes of associative learning. The processes of response learning and associative learning (see Underwood \& Schultz, 1960) are well established and are important in the current model. Response learning refers to the establishment of the availability of responses qua responses, as demonstrated by overt evocation regardless of the correctness of responding. Response learning comprises the two possible components of response integration (the association into a unitary response of poorly integrated, unfamiliar items such as CCC trigrams) and response selection (the selection of list-responses from within a total response repertoire). Response learning is measured by the trial of first evocation for a given item. Associative learning refers to the acquisition of specific stimulusresponse connections and is measured by the additional number of trials from response learning to some criterion of correct responding (see Kanak \& Nuener, 1970). Although accurate measurement of these two stages may be affected by differential response-confidence thresholds produced by the presence of intratask competition (Ekstrand, 1966), the application to single-list PA learning of noncompetitive materials can be made as a reasonably precise test of the model.
The present model is similar to the Rescorla and Wagner (1972) model in that relationships between variables are expressed through mathematical formulas. However, as in the Rescorla-Wagner model, the actual numerical values of the variables are not predicted; rather, the manner in which the variables interact is predicted. The present model is more "Mackintoshian" than "RescorlaWagnerian" in theoretical orientation, however, in that the present model allows for variability in the saliency of the stimuli, an issue that Mackintosh (1975) addressed, although Rescorla and Wagner did not. In fact, it is this predicted variability in the saliency of the contextual stimuli that is of primary concern within the current model. Specifically, the theory states that under conditions wherein the environmental context is indicative of differential reinforcement, contextual cues may, over trials, acquire sufficient saliency that they become capable of eliciting response items.

The following equation is proposed to account for the role of environmental context on response integration:

$$
\alpha_{\mathrm{c}}=\frac{1}{\lambda+\mathrm{I}_{\mathrm{x}}}+\beta
$$

where

$$
\alpha_{c}=\text { saliency of the environmental context }
$$

$\lambda=$ asymptotic level of response item or response list meaningfulness. Range is from an arbitrarily small decimal to 1.00 . Within experimental constraints it is not reasonable to assume that "DOG" and "QPR" will acquire equivalent degrees of meaningfulness.

$\mathrm{I}_{\mathbf{x}}=$ total amount of integration present on trial $\mathbf{n}$. Range $=0.00-1.00$.

$\beta=$ factors external to the materials employed that affect the acquisition rate, such as age, fatigue, various personality variables, individual differences in response-confidence thresholds, presentation rate, and method or mode of presentation.

This formulation predicts that as response meaningfulness increases and therefore preexperimental integration also increases, the role of context as a potent contributor to response integration will be mitigated. Conversely, the role of context within response integration will increase if responses are initially of low meaningfulness and are preexperimentally poorly integrated.

Except in the case in which $I_{x}=1.00, I_{x}$ will change from trial to trial. Such a deduction provides divergent predictions for the effect of degree of learning (DOL) on $\alpha_{\mathrm{c}}$. That is, with low DOL, the effects of context should be greater than at higher DOL, at which subjects are not only perceiving the response item as an integrated whole, but are also establishing the stimulus-response (S-R) bond and perhaps also utilizing "higher or deeper levels" of processing (Craik \& Lockhart, 1972) in the formation of the specific association.

The saliency of the context $\left(\alpha_{c}\right)$ is assumed to be an 
inverse function of $\lambda$ and $I_{x}$. This inverse relationship is predicted because (1) as response integration $\left(I_{\mathbf{x}}\right)$ nears completion over trials and thus becomes a less potent factor in the PA task, the saliency of the cues for integration should also diminish, and (2) the greater the $\lambda$, the less important is the response integration stage to the completion of the PA task. Thus, if $\lambda$ is relatively high, both the role of response integration and the saliency of the contextual cues for integration should be relatively low.

$\beta$ has an additive rather than a multiplicative function in the model. This function is intentionally utilized to provide a bypass for the situation in which both $\lambda$ and $I_{x}$ are close to zero and thereby allows $\alpha_{c}$ to contribute to the learning process even when the remainder of the equation might be functionally zero. This specification results in the prediction that if response meaningfulness is functionally zero, $I_{\mathbf{x}}$ is zero, and $\beta$ is high (via the instructions, etc.), contextual cues will, for example, because of the importance of the task, still continue to function as sources of association. Similarly, individuals with certain personality characteristics such as high field dependency may have a "natural" and greater propensity to attend to (at some level of consciousness) or be affected by context-item associations regardless of stimulus/response characterics.

The role of meaningfulness produces antithetical effects on context-item associations in response selection, relative to its effects upon response integration. The proposed deduction for this stage is:

$$
\alpha_{\mathrm{c}}=\left|\lambda-\mathbf{R}_{\mathbf{I}}+\left(-\mathbf{R}_{\mathbf{S}}\right)\right|+\beta,
$$

where $\alpha_{\mathrm{c}}, \lambda$, and $\beta$ retain their previous definitions, and

$\mathbf{R}_{\mathbf{I}}=$ the amount of within-experiment response integration required for a particular response or response list to be fully integrated. Range = $0.0-1.00$.

$R_{S}=$ degree of formal or semantic similarity between response items within a list. When items within a list have no semantic associations and only minimal formal similarily, then $R_{s}=0.0$. On the other hand, $R_{S}=1.0$ when all responses within a list are identical. For most situations, excluding mixed-list designs, the impact of $\mathbf{R}_{\mathbf{S}}$ will be only minimal.

It is important to realize that the absolute value $\left|\lambda-R_{I}+\left(-R_{S}\right)\right|$, rather than the "real" value, is employed. The absolute value is utilized to avoid subtracting from $\beta$, which is affected by variables such as instructions and personality variables rather than specific characteristics of the items. Also, note that $\mathbf{R}_{\mathbf{I}}$ is subtracted from $\lambda$, since the amount of response integration required should reduce the importance of $\lambda$ in determining the overall importance of response selection in the PA task.
$R_{S}$ is subtracted from $\lambda-R_{I}$ because, as semantic or formal similarity of responses within a list increases, subjects will be more likely to utilize available mnemonic cues or functional stimuli that will override the influence of elicited contextual associations.

Finally, consider the application of such a model to the associative learning stage. An inverse or indirect relationship between $\alpha_{\mathrm{c}}$ and S-R meaningfulness is assumed in the associative stage. Specifically,

$$
\alpha_{\mathrm{c}}=\left(\lambda_{\mathrm{AL}}-\left|\mathrm{V}_{\mathrm{SR}}\right|\right)+\beta,
$$

where $\beta$ and $\alpha_{\mathrm{c}}$ retain their previous definitions and

$\lambda_{\mathrm{AL}}=\mathrm{a}$ function of preexperimental learning and equals the total amount of associative strength that a given association can accrue; range $=$ an arbitrarily small decimal to 1.00 . Thus, the pair DOG-CAT is assumed to have a higher $\lambda_{\mathrm{AL}}$ than, for example, TRV-CFQ because of subjects' preexperimental experience.

$V_{S R}=$ the amount of associative strength between the stimulus and response present on any one trial; range $=0.00 \pm 1.00$. $V_{S R}$ is similar to $I_{x}$ in that it changes from trial to trial and thus is a function of the number of learning trials. $V_{S R}$ acquires a negative value in situations, for example, in which the primary associates of the stimulus items are in the response list but, rather than being paired with their respective associatively related stimuli, are paired with another stimulus.

The form of the equation is rather straightforward for this stage: (1) $\beta$ has an additive function due to its independence from list materials; (2) $\alpha_{\mathrm{c}}$ will be greater when $V_{S R}=0.0$ than when it is either positive or negative; (3) $V_{S R}$ is subtracted from $\lambda_{A L}$, since it is assumed that, as intrapair associative strength increases, it will approach $\lambda$ and thus reduce and override the saliency of contextual cues as other forms of associative learning (e.g., semantic networks) become available.

The last point is important to the essence of the present model. Specifically, it is assumed that if other forms of associative learning, particularly those involving obvious semantic or phonemic associations, are available to the subjects, the influence of environmental context will be mitigated.

As indicated previously, the current model provides predictive power, as well as functioning as an explanatory and descriptive tool, a combination of functions that previous explanations have not provided. The present theoretical proposal has received initial empirical support (Nixon, 1982), although such data are not provided in this paper due to page constraints. It is important to remember that the model is a theoretical analogue of the processes involved in classical conditioning. Furthermore, the formulas are simply a means of expressing the proposed relationships between the variables involved in one 
type of learning, that is, PA learning. The theoretical implications involving the elicitation of retrieval processes are much broader and apply to a broad range of other learning and memory tasks. In summary, the present theoretical model is a major advancement in our understanding of the environmental context effect because it (1) provides a theoretical explanation taking into account existing empirical data, (2) allows specific predictions which include accounting for those situations in which " different" contexts for learning two tasks produce superior learning and retention, (3) is esthetically pleasing, and (4) provides a framework for future research development.

\section{REFERENCES}

Bilodeau, I. M., \& Schlosberg, H. (1951). Similarity in stimulating conditions as a variable in retroactive inhibition. Journal of Experimental Psychology,41, 199-204.

BLoCK, R. A. (1982). Temporal judgments and contextual change. Journal of Experimental Psychology: Learning, Memory, and Cognition, 8, 530-544.

Craik, F. I. M., \& LockHART, R. S. (1972). Levels of processing: A framework for memory research. Journal of Verbal Learning and Verbal Behavior, 11, 671-684.

Dallett, K., \& Wilcox, S. G. (1968). Contextual stimuli and proactive inhibition. Journal of Experimental Psychology, 78, 475-480.

Eckert, E., KanaK, N. J., \& STevens, R. (1984). Memory for frequency as a function of the environmental context. Bulletin of the Psychonomic Society, 22, 507-510.

EKSTRAND, B. R. (1966). A note on measuring response learning during paired-associate learning. Journal of Verbal Learning and Verbal Behavior, 5, 344-347.

GodDEN, D. R., \& BADDEley, A. D. (1975). Context-dependent memory in two natural environments: On land and underwater. British Journal of Psychology, 66, 325-331.

GreENSPOON, J., \& RANYARD, R. (1957). Stimulus conditions and retroactive inhibition. Journal of Experimental Psychology, 53, 55-59.

Hintzman, D. L., Block, R. A., \& Summers, J. J. (1973). Contextual associations and memory for serial position. Journal of Experimental Psychology, 97, 220-229.
Jobe, J. B., Mellgren, R. L., Feinberg, R. A., Littlejohn, R. L., \& RIGBY, R. L. (1977). Patterning, partial reinforcements, and Nlength effects at spaced trials as a function of reinstatement of retrieval cues. Learning and Motivation, 8, 77-97.

KanaK, N. J., \& Neuner, S. D. (1970). Associative symmetry and item availability as a function of five methods of paired-associate acquisition. Journal of Experimental Psychology, 86, 288-295.

MACKINTOSH, N. J. (1975). A theory of attention: Variations in the associability of stimuli with reinforcement. Psychological Review, 52, 276-298.

McGeOCH, J. A. (1942). The psychology of human learning. New York: Longmans.

MCGoverN, J. B. (1964). Extinction of associations in four transfer paradigms. Psychological Monographs, 78(16, Whole No. 593).

Nixon, S. J. (1982). Environmental cues and associative learning. Unpublished doctoral dissertation, University of Oklahoma.

NIXON, S. J., \& KANAK, N. J. (1981). The interactive effects of instructional set and environmental context changes on the serial position effect. Bulletin of the Psychonomic Society, 18, 237-240.

Postman, L., \& UNDERWood, B. J. (1973). Critical issues in interference theory. Memory \& Cognition, 1, 19-40.

Rescorla, R. A., \& WAgneR, A. W. (1972). A theory of Pavlovian conditioning: Variations in the effectiveness of reinforcement and nonreinforcement. In A. H. Black \& W. F. Prokasy (Eds.), Classical conditioning II: Current research and theory. New York: AppletonCentury-Crofts.

Smith, S. M. (1979). Remembering in and out of context. Journal of Experimental Psychology: Human Learning and Memory, 5, 460-471.

SMITH, S. M. (1982). Enhancement of recall using multiple environmental contexts during learning. Memory \& Cognition, 10, 405-412.

SMith, S. M., GlenberG, A., \& BJorK, R. A. (1978). Environmental context and human memory. Memory \& Cognition, 6, 343-353.

UNDERWOOD, B. J., \& SCHULTZ, R. W. (1960). Meaningfulness and verbal learning. Philadelphia: Lippincott.

WiCKens, C., Tuber, D. S., \& WiCKENS, D. D. (1983). Memory for the conditioned response: The proactive effect of preexposure to potential conditioning stimuli and context change. Journal of Experimental Psychology: General, 112, 41-57.

(Manuscript received for publication October 1, 1984.) 